\title{
Mode structure symmetry breaking of energetic particle driven Beta-induced Alfvén Eigenmode
}

\author{
Z. X. Lu, ${ }^{1, a)}$ X. Wang, ${ }^{1}$ P. Lauber, ${ }^{1}$ and F. Zonca ${ }^{2,3}$ \\ 1) Max Planck Institut für Plasmaphysik, Garching, Germany \\ ${ }^{2)}$ ENEA C.R. Frascati, Via Enrico Fermi 45, CP 65-00044 Frascati, Italy \\ ${ }^{3)}$ Institute for Fusion Theory and Simulation and Department of Physics, Zhejiang University, Hangzhou 310027, \\ People's Republic of China
}

The mode structure symmetry breaking of energetic particle driven Beta-induced Alfvén Eigenmode (BAE) is studied based on global theory and simulation. The weak coupling formula gives reasonable estimate of the local eigenvalue compared with global hybrid simulation using XHMGC. The non-perturbative effect of energetic particles on global mode structure symmetry breaking in radial and parallel (along $\mathbf{B}$ ) direction is demonstrated. With the contribution from energetic particles, two dimensional (radial and poloidal) BAE mode structures with symmetric/asymmetric tails are produced using an analytical model. It is demonstrated that the symmetry breaking in radial and parallel direction is intimately connected. The effects of mode structure symmetry breaking on nonlinear physics, energetic particle transport and the possible insight for experimental studies are discussed.

\section{INTRODUCTION AND MOTIVATION}

In non-uniform tokamak plasmas, the $2 \mathrm{D}$ mode structure (in radial and poloidal directions) is related to the wave-particle interaction and the consequent instability excitation and energetic particle transport ${ }^{1-4}$. Symmetry breaking of the $2 \mathrm{D}$ mode structure can also be important for understanding momentum transport, specifically, the intrinsic momentum transport ${ }^{5,6}$. While numerical simulations based on initial value approach or eigenvalue approach have been used to study energetic particle problems (see, e.g., Ref [7-9]), other theoretical approaches have also been developed ${ }^{10,11}$. The generalized fishbone-like dispersion relation (GFLDR $)^{1-3}$ using the Mode Structure Decomposition (MSD) method ${ }^{11}$ provides a general framework for the study of Alfvén wave and energetic particle physics. In this work, we focus on the important role of global mode structure, in particular, the radial and parallel symmetry breaking in terms of $k_{r}$ and $k_{\| \mid}$for weakly coupled poloidal harmonics. For weak magnetic shear $(\hat{s})$ and low toroidal mode number $(n)$, the coupling between poloidal harmonics is weak as shown in our work, in contrast to that for high $n$, moderate to high $\hat{s}$ micro turbulence. The non-perturbative effect of energetic particles on mode frequency and structure are studied and its connection to the nonlinear dynamics is discussed. The purpose of this work includes, but is not limited to the following aspects.

1. While global theory has been developed for BAE to demonstrate the "boomerang" shape structure due to the anti-Hermitian contribution of energetic particles using GFLDR ${ }^{12}$, this work provides a complementary approach in weak coupling limit, by using the original gyrokinetic quasi-neutrality and vorticity equations ${ }^{13}$ for few coupled poloidal harmonics.

\footnotetext{
a) Electronic mail: zhixin.lu@ipp.mpg.de
}

2. By focusing on the weak coupling case, this work, with energetic particles and electromagnetic physics taken into account, serves as a complement of our previous symmetry breaking study for micro-turbulence ${ }^{14}$ (strong coupling case, where many poloidal harmonics are coupled to form the global mode structure). The weak coupling formulae can also be useful for the study of betainduced Alfvén-acoustic eigenmode (BAAE) where the observed mode structure from experiment and gyrokinetic studies is dominant by a single ploidal harmonic $^{15-17}$.

3. This work can shed light on the understanding of the mode structure excited by energetic particles $(\mathrm{EPs})^{18}$ and the reasons of the different behaviors of the co-passing and counter-passing $\operatorname{EPs}^{19,20}$.

4. More generally, by identifying the basic ingredient of symmetry breaking of mode structure, this work can provide an input to other perturbative/nonperturbative codes and suggest interpretation of simulation results ${ }^{7,21-23}$.

This work is organized as follows. In Section II, the physics model and the theoretical approach for the energetic particle driven BAE are introduced. In Section III, the theoretical analyses and the simulation results are given along with the symmetry breaking studies in terms of $k_{r}$ and $k_{\|}$. In Section IV, we give the discussion and outlook.

\section{PHYSICS MODEL}

\section{A. Gyrokinetic quasi-neutrality and vorticity equations with kinetic thermal ion and energetic particle}

In this work, we use the equations for the perturbed scalar potential $\delta \phi$, the perturbed field $\delta \psi$, which is related to the parallel vector potential fluctuation $\delta A_{\|}$by 
$\delta A_{\|} \equiv-i(c / \omega) \mathbf{b} \cdot \nabla \delta \psi$, and the non adiabatic perturbed particle distribution function $\delta K_{s}$, which is related to the perturbed particle distribution function $\delta f_{s}$ by

$\delta f_{s}=\frac{e_{s}}{m_{s}}\left[\frac{\partial F_{0, s}}{\partial \epsilon} \delta \phi-J_{0}\left(a_{s}\right) \frac{Q F_{0 s}}{\omega} \delta \psi e^{i L_{k s}}\right]+\delta K_{s} e^{i L_{k s}}$

$L_{k s}=\left(m_{s} c / e_{s} B\right)(\mathbf{k} \times \mathbf{b} \cdot \mathbf{v}), \epsilon=v^{2} / 2^{13,24}$. The right handed coordinate system $(r, \theta, \phi)$ and $(R, Z, \phi)$ are used where $r, \theta$ and $\phi$ are radial-like, poloidal-like and toroidal-like coordinates. The subscripts $s=i, e, f$ indicate thermal ions, electrons and fast (energetic) particles respectively. The toroidal current and toroidal magnetic field are both in $\phi>0$ direction. Then the electromagnetic system is described by the gyrokinetic equation (GKE), vorticity equation (VE) and quasi-neutrality equation (QNE) as follows ${ }^{13}$,

$$
\begin{aligned}
& {\left[v_{\|} \partial_{\|}-i\left(\omega-\omega_{d}\right)\right]_{s} \delta K_{s}=i \frac{e_{s}}{m_{s}} Q F_{0 s}\left[J_{0}\left(a_{s}\right)(\delta \phi-\delta \psi)+\frac{\omega_{d s}}{\omega} J_{0}\left(a_{s}\right) \delta \psi\right]} \\
& B \partial_{\|}\left[\frac{1}{B} \frac{k_{\perp}^{2}}{k_{\theta}^{2}} \partial_{\|} \delta \psi\right]+\frac{\omega^{2}}{v_{A}^{2}}\left(1-\frac{\omega_{* p i}}{\omega}\right) \frac{k_{\perp}^{2}}{k_{\theta}^{2}} \delta \phi+\frac{\alpha}{q^{2} R^{2}} g \delta \psi=\left\langle\sum_{s=i, f} \frac{4 \pi e_{s}}{c^{2} k_{\theta}^{2}} J_{0}\left(a_{s}\right) \omega \omega_{d s} \delta K_{s}\right\rangle \\
& \left(1+\frac{1}{\tau}\right)(\delta \phi-\delta \psi)+\left(1-\frac{\omega_{* p i}}{\omega}\right) b_{i} \delta \psi=\frac{T_{i}}{n e}\left\langle J_{0}\left(a_{i}\right) \delta K_{i}\right\rangle
\end{aligned}
$$

where $\omega_{d, s}=v_{d, s} k_{\theta} g, v_{d, s}=-\left(v_{\|}^{2}+v_{\perp}^{2} / 2\right) /\left(\omega_{c, s} R\right)$, $g=\cos \theta+\left(k_{r} / k_{\theta}\right) \sin \theta, \omega_{c s}=e_{s} B /\left(m_{s} c\right), Q F_{0 s}=$ $\omega\left(\partial F_{0 s} / \partial \epsilon\right)+\left(1 / \omega_{c s}\right) \mathbf{k} \times \mathbf{b} \cdot \nabla F_{0 s}, \quad b_{s}=k_{\perp}^{2} \rho_{t s}^{2} / 2$, $\rho_{t s}=c m_{s} v_{t s} /\left(e_{s} B\right), v_{t s}=\sqrt{2 T_{s} / m_{s}}, \alpha=-R_{0} q^{2} \beta^{\prime}$, $\beta=8 \pi P / B^{2}, \quad P$ is the total plasma pressure, $\omega_{* p s}=\omega_{* s}+\omega_{* T s}, \omega_{* s}=-\rho_{t s} v_{t s} k_{\theta} /\left(2 L_{n s}\right), \omega_{* T s}=$ $-\rho_{t s} v_{t s} k_{\theta} /\left(2 L_{T s}\right), \quad L_{n s}=-\left(d \ln n_{s} / d r\right)^{-1}, \quad L_{T s}=$ $-\left(d \ln T_{s} / d r\right)^{-1}, \tau=T_{e} / T_{i}$, and $\langle\ldots\rangle$ indicates the integral in velocity space. Here, $J_{0}\left(a_{s}\right)$ accounts for finite Larmor radius effects using the standard notation for the
Bessel function and $a_{s}=k_{\perp} \rho_{s}$. Furthermore, for simplicity, we assume high aspect-ratio, low-pressure tokamak plasma equilibrium with concentric circular magnetic flux surfaces.

In order to obtain the eigenvalue equation, firstly, $\delta K=\delta K(\Delta \phi, \delta \psi)$ is obtained from GKE where $\Delta \phi \equiv$ $\delta \phi-\delta \psi$; secondly, $\delta K(\Delta \phi, \delta \psi)$ is substituted into the QNE to obtain $\Delta \phi=\Delta \phi(\delta \psi)$; and lastly, $\delta K(\delta \psi)$ and $\Delta \phi(\delta \psi)$ are substituted into the VE to obtain the linearized eigenvalue equation. With Fourier decomposition $\delta y=\delta y_{n} \exp \{-i n \phi\}$ where $\delta y=\delta \psi, \delta \phi, \delta K$ and integration along un-perturbed particle orbit, Eqs. 1 and 3 yield

$$
\begin{aligned}
J_{0}\left(a_{i}\right) \delta K_{i, n} & =\frac{e}{m_{i}} \frac{Q F_{0 i}}{w} J_{t, i}\left\{-\frac{\left(1-\omega_{* p i} / \omega\right) b_{i} \delta \psi_{m}}{\bar{D}_{m} R_{m, i}}-\frac{\delta_{a t, i} \bar{N}_{m} \delta \psi_{m-1}}{\bar{D}_{m} R_{m, i}}-\frac{\delta_{b t, i} \bar{N}_{m} \delta \psi_{m+1}}{\bar{D}_{m} R_{m, i}}+\frac{\delta_{a, i} \delta \psi_{m-1}}{R_{m, i}}+\frac{\delta_{b, i} \delta \psi_{m+1}}{R_{m, i}}\right\} e^{i m \theta} \\
J_{0}\left(a_{f}\right) \delta K_{f, n} & =\frac{e}{m_{f}} \frac{Q F_{0 f}}{w} J_{t, f}\left\{\frac{\delta_{a, f} \delta \psi_{m-1}}{R_{m, f}}+\frac{\delta_{b, f} \delta \psi_{m+1}}{R_{m, f}}\right\} e^{i m \theta},
\end{aligned}
$$

where $R_{m, s}=\bar{v}_{\|, s} / \zeta_{m, s}-1, \bar{v}_{\|}=v_{\|} / v_{t s}, \zeta_{m, s}=$ $\Omega^{s} /(m-n q), \Omega^{s} \equiv \omega / \omega_{t s}, \omega_{t s}=v_{t s} /(q R), \delta_{a / b, s}=$ $v_{d, s} k_{a / b} / \omega, \delta_{a t / b t, s}=v_{d t, s} k_{a / b} / \omega, v_{d t, s}=-v_{t s}^{2} /\left(\omega_{c, s} R\right)$, $k_{a / b}=\left(k_{\theta} \mp i k_{r}\right) / 2, k_{\perp}^{2}=k_{r}^{2}+m^{2} / r^{2}, v_{t s}=\sqrt{2 T_{s} / m_{s}}$, $\bar{D}_{m, s} \equiv \bar{D}_{s}\left(\zeta_{m, s}\right)=1+1 / \tau+\left\langle\kappa_{s} \bar{F}_{0 s} J_{t, s} / R_{m, s}\right\rangle, \bar{F}_{o s}=$ $F_{0 s} / n_{0 s}, \quad J_{t, s}=J_{0}^{2}\left(a_{s}\right) J_{0}^{2}\left(\lambda_{s}\right), \quad \lambda_{s}=-q R v_{d, s} k_{\perp} / v_{\|}$, $\kappa_{s}=-\left(v_{t s}^{2} / 2\right)\left(Q F_{0 s} / \omega\right) / F_{0 s}$, repeated subscripts indicate summation. For the sake of simplicity, in the following, the subscript ' $\mathrm{s}$ ' in $\zeta_{m, s}, R_{m, s}$ etc is omitted when no ambiguity is brought in. In deriving $\delta K_{f, n}$, only the dominant kinetic particle compression (KPC) contribution is kept for the parameters in Table $\mathrm{I}\left(k_{\perp} \rho_{f, b} \approx 0.092\right.$ for EP birth energy, $n=2$ and normalized EP density $\left.n_{f} / n_{i} \ll 1\right)$. Since we focus on the study of low $n$ mode, only $J_{0}(\lambda)$ is kept for finite orbit width (FOW) effect. Note, however, that the present choice of mode number does not correspond to the most unstable mode and that, unlike the typical conditions discussed in Ref. [3], the EP response is dominated by the fine radial structure in the kinetic/singular layer ${ }^{1,2}$. With Eqs. 4, and 5 substituted into 2, by defining $K P C_{s} \equiv\left\langle 4 \pi e_{s} /\left(c^{2} k_{\theta}^{2}\right) J_{0}\left(a_{s}\right) \omega \omega_{d s} \delta K_{s}\right\rangle$, we have 


$$
\begin{aligned}
K P C_{i}= & -\frac{2 v_{t i}^{2}}{k_{\theta}^{2} v_{A}^{2} R^{2}}\left\{-\left(1-\frac{\omega_{* p i}}{\omega}\right) b_{i} \frac{\bar{N}_{m-1}}{\bar{D}_{m-1}} \frac{k_{a} \omega}{v_{d t, i}} \delta \psi_{m-1}-\left(1-\frac{\omega_{* p i}}{\omega}\right) b_{i} \frac{\bar{N}_{m+1}}{\bar{D}_{m+1}} \frac{k_{b} \omega}{v_{d t, i}} \delta \psi_{m+1}\right. \\
& -k_{a}^{2} \frac{\bar{N}_{m-1}^{2}}{\bar{D}_{m-1}} \delta \psi_{m-2}-k_{a} k_{b} \frac{\bar{N}_{m+1}^{2}}{\bar{D}_{m+1}} \delta \psi_{m}-k_{a} k_{b} \frac{\bar{N}_{m-1}^{2}}{\bar{D}_{m-1}} \delta \psi_{m}-k_{b}^{2} \frac{\bar{N}_{m+1}^{2}}{\bar{D}_{m+1}} \delta \psi_{m+2} \\
& \left.+k_{a}^{2} \bar{H}_{m-1, i} \delta \psi_{m-2}+k_{a} k_{b} \bar{H}_{m+1, i} \delta \psi_{m}+k_{a} k_{b} \bar{H}_{m-1, i} \delta \psi_{m}+k_{b}^{2} \bar{H}_{m+1, i} \delta \psi_{m+2}\right\}, \\
K P C_{f}= & -\frac{2 v_{t i}^{2} \bar{n}_{f}}{k_{\theta}^{2} v_{A}^{2} R^{2}}\left\{k_{a}^{2} \bar{H}_{m-1, f} \delta \psi_{m-2}+k_{a} k_{b} \bar{H}_{m+1, f} \delta \psi_{m}+k_{a} k_{b} \bar{H}_{m-1, f} \delta \psi_{m}+k_{b}^{2} \bar{H}_{m+1, f} \delta \psi_{m+2}\right\},
\end{aligned}
$$

where $\bar{n}_{f}=n_{f} / n_{i}, \bar{N}_{m} \equiv \bar{N}\left(\zeta_{m, i}\right)=\left\langle J_{t, i} \kappa_{i} \bar{F}_{0 i}\left(\bar{v}_{\perp}^{2} / 2+\right.\right.$ $\left.\left.\bar{v}_{\| \mid}^{2}\right) / R_{m, i}\right\rangle, \quad \bar{H}_{m, s} \equiv \bar{H}\left(\zeta_{m, s}\right)=\left\langle J_{t, s} \kappa_{s} \bar{F}_{0 s}\left(\bar{v}_{\perp}^{2} / 2+\right.\right.$ $\left.\left.\bar{v}_{\mid l}^{2}\right)^{2} / R_{m, s}\right\rangle$. The response functions $\bar{D}_{m, s}, \bar{N}_{m, s}$ and $\bar{H}_{m, s}$ describe the response of $\delta n_{s}$ (density perturbation) and $\delta j_{s}$ (current perturbation) to $\Delta \phi$ and $\delta \psi$.

For thermal ion with Maxwellian distribution $\bar{F}_{0 i}=\exp \left\{-\bar{v}^{2}\right\} /\left(\pi^{3 / 2} v_{t i}^{3}\right)$, we have $\kappa_{i}=1-\omega_{* i} / \omega\left[1+\eta_{i}\left(\bar{v}^{2}-3 / 2\right)\right]$ and $\bar{H}_{i, m}=$ $\left(1-\omega_{* i} / \omega\right) \bar{F}_{i, m}-\omega_{* T i} \bar{G}_{i, m}$. By ignoring the finite Larmor radius (FLR) and finite orbit width (FOW) effects $\left(J_{t}=1\right)$, which is valid for parameters in Table I $\left(k_{\theta} \rho_{t i} \approx 0.0226\right), \bar{D}_{m}, \bar{N}_{m}, \bar{F}_{m}$ and $\bar{G}_{m}$ can be calculated analytically and are equivalent to $D, N, F$ and $G$ in [13] and [25] with the connection $N(\zeta)=\bar{N}(\zeta) / \zeta$, $F(\zeta)=\bar{F}(\zeta) / \zeta, G(\zeta)=\bar{G}(\zeta) / \zeta$ and $D(\zeta)=\bar{D}(\zeta) / \zeta$ $(D, N, F$ and $G$ are variables defined in [13]), where $\bar{D}=1+\frac{1}{\tau}+\left(1-\frac{\omega_{*}}{\omega}\right) \zeta Z(\zeta)-\frac{\omega_{* T}}{\omega} \zeta\left[\zeta+\left(\zeta^{2}-\frac{1}{2}\right) Z(\zeta)\right]$, $\bar{N}=\zeta\left(1-\frac{\omega_{*}}{\omega}\right)\left[\zeta+\left(\zeta^{2}+\frac{1}{2}\right) Z(\zeta)\right]-\zeta \frac{\omega_{* T}}{\omega}\left[\zeta\left(\zeta^{2}+\frac{1}{2}\right)+\right.$ $\left.\left(\zeta^{4}+\frac{1}{4}\right) Z(\zeta)\right], \bar{F}=\zeta^{2}\left(\zeta^{2}+\frac{3}{2}\right)+\zeta\left(\zeta^{4}+\zeta^{2}+\frac{1}{2}\right) Z(\zeta)$, $\bar{G}=\zeta^{2}\left(\zeta^{4}+\zeta^{2}+2\right)+\zeta\left(\zeta^{6}+\frac{\zeta^{4}}{2}+\zeta^{2}+\frac{3}{4}\right) Z(\zeta)$ and $Z(\zeta)=(1 / \sqrt{\pi}) \int_{-\infty}^{\infty} d x e^{-x^{2}} /(x-\zeta)$ is the plasma dispersion function. Based on the multiple spatial scales of the fluctuation structure, the analyses in this work is equivalent to the asymptotic matching approach ${ }^{1-3}$. In the following, we focus on the BAE mode with $\omega / \omega_{t i} \gg 1$. Then for weak coupling case, the dominating poloidal harmonic, referred to as "central poloidal harmonic" in the following, has much larger amplitude than its neighboring ones (cf. Fig. 5 of [26]). By keeping only the central poloidal harmonics, Eq. 6 yields

$$
K P C_{i}=\frac{k_{\perp}^{2} \omega_{B A E}^{2}}{k_{\theta}^{2} v_{A}^{2}} \delta \psi_{m} e^{i m \theta}
$$

where $\omega_{B A E}^{2}=(7 / 4+\tau) v_{t i}^{2} / R^{2}$.

For energetic particles, we assume a slowing down distribution ${ }^{27}$,

$$
F_{f}=\frac{C_{0} \delta\left(\Lambda-\Lambda_{0}\right)}{\epsilon^{3 / 2}} H\left(\epsilon_{b}-\epsilon\right)
$$

where $C_{0}=\sqrt{2\left(1-\Lambda_{0} B\right)} B \beta_{f} /\left(2^{5} \pi^{2} m_{f} \epsilon_{b}\right), \delta(x)$ is the Dirac delta function, the pitch angle is defined as $\Lambda=$ $v_{\perp}^{2} /\left(v^{2} B\right), \epsilon_{b}$ is the birth energy, the Heaviside function $\vec{H}(x)=0$ for $x<0$ and $H(x)=1$ for $x>0$, the EP beta $\beta_{f}=4 \pi \int d v^{3} F_{f} m v^{2} / B^{2}$. Then for weak coupling case, Eq. 7 yields

$$
\begin{aligned}
K P C_{f} & =2 \frac{\bar{n}_{f} v_{t f}^{2}}{k_{\theta}^{2} v_{A}^{2} R^{2}} \frac{\omega_{* f}}{\omega} \times \frac{\left(1-\Lambda_{0} B / 2\right)^{2}}{1-\Lambda_{0} B} \delta \psi_{m} e^{i m \theta} \\
& \times k_{a} k_{b} \sum_{\sigma=m \pm 1}\left\{\zeta_{\sigma, f}^{2}\left[1+\bar{\zeta}_{\sigma, f}^{2} \ln \left(1-\frac{1}{\bar{\zeta}_{\sigma, f}^{2}}\right)\right]\right\},
\end{aligned}
$$

where $\bar{\zeta}_{\sigma, f}=\zeta_{\sigma, f}^{2} /\left[2\left(1-\Lambda_{0} B\right) \bar{\epsilon}_{b}\right]$, uniform EP temperature profile is assumed and only EP density is considered to be non-uniform.

\section{B. Global and local analysis of BAE eigenvalue and mode structure}

With Eqs. 8 and 10 substituted into Eq. 2, the global equation for uniform thermal ion is obtained

$$
\begin{aligned}
B \partial_{\|}\left[\frac{\nabla_{\perp}^{2} \partial_{\|} \delta \psi}{B}\right]+\nabla \cdot\left[\frac{\omega^{2}-\omega_{B A E}^{2}}{v_{A}^{2}} \nabla_{\perp} \delta \psi\right] & +k_{\theta}^{2} K P C_{f} \\
=0 & ,
\end{aligned}
$$

where $K P C_{f}$ includes the non-Hermitian part due to EPs. In large aspect ratio and weak coupling limit, Eq. 11 reduces to

$$
Y_{0} \frac{\partial^{2}}{\partial r^{2}} \delta \psi_{m}+Y_{1} \frac{\partial}{\partial r} \delta \psi_{m}+Y_{2} \delta \psi_{m}=0
$$

where $Y_{0}=-k_{\|}^{2}+\left(\omega^{2}-\omega_{B A E}^{2}+\omega_{t i}^{2} \overline{K P C}_{f, m}\right) / v_{A}^{2}$, $Y_{1}=-2 k_{\| \mid} \partial k_{\|} / \partial r, Y_{2}=-\left[Y_{0}(r) k_{\theta}^{2}+k_{\| \mid} \partial^{2} k_{\| \mid} / \partial r^{2}\right]$, and $\overline{K P C}_{f, m} \equiv K P C_{f, m}\left[\omega_{t i}^{2} /\left(k_{\theta}^{2} v_{A}^{2}\right) k_{\perp}^{2} \delta \psi_{m}\right]^{-1}$. In deriving Eq. 12, we ignored $O(r / R)$ terms from $\left(\nabla v_{A}\right) / v_{A}$ and $\nabla n_{i} / n_{i}$ terms due to the large aspect ratio assumption and the uniform thermal ion assumption; we ignored the variation of the metric tensor along B, i.e., $\partial_{\|} \nabla_{\perp}^{2}=0$, (but $\nabla_{\perp}^{2} \partial_{\|}=i \nabla_{\perp}^{2} k_{\|} \neq 0$ ); we used $\nabla_{\perp}^{2} \approx$ $(1 / r) \partial / \partial r(r \partial / \partial r)+\left(1 / r^{2}\right) \partial^{2} / \partial \theta^{2}$.

The local dispersion relation for BAE is obtained in 
weak coupling limit that corresponds to $Y_{0}=0$, i.e.,

$$
\begin{aligned}
D_{0} & =\Omega^{2}-\Omega_{B A E}^{2}-\frac{(n q-m)^{2}}{\beta_{i}}-\bar{C}_{f} \frac{\left(1-\Lambda_{0} B / 2\right)^{2}}{1-\Lambda_{0} B} \frac{\Omega}{2} \\
& \times \sum_{\sigma=m \pm 1} \frac{1}{(\sigma-n q)^{2}}\left[1+\bar{\zeta}_{\sigma, f}^{2} \ln \left[1-\frac{1}{\bar{\zeta}_{\sigma, f}^{2}}\right]\right]=0,
\end{aligned}
$$

where the frequency is normalized with $\omega_{t i}=v_{t i} /(q R)$, $\Omega_{B A E}^{2}=(7 / 4+\tau) q^{2}, \bar{C}_{f}=-\bar{n}_{f} \tau_{f i} k_{\theta} \rho_{i} q^{3} /\left(2 \bar{e}_{f} \varepsilon_{n f}\right)$, $\bar{e}_{f}=e_{f} / e_{i}, \tau_{f i}=T_{f} / T_{i}, 1 / \varepsilon_{n f}=R / L_{n f}$. Note that while the local solution corresponds to a small radial scale $\left(\left|k_{r} L_{e q}\right| \gg 1\right.$, where $L_{e q}$ is the equilibrium characteristic length), EPs are still dominated by the geodesic curvature response to provide the instability drive. Namely, $k_{r} \rho_{f}(n=2)$ is assumed to be small so that the deviation of $J_{0}\left(\lambda_{f}\right)$ away from 1 is small. This is valid as long as the radial mode width $\left(\sim 1 / k_{r}\right)$ is in the mediate spatial scale between $\rho_{f}$ and $L_{e q}$. As shown in Section III, this gives a reasonable estimate of growth rate for our considered low $n$ mode. At the mode rational surface $(n q-m=0), \zeta_{ \pm}= \pm \Omega^{f}=\omega / \omega_{t f}$, and

$$
\begin{gathered}
D_{0}=\Omega^{2}-\Omega_{B A E}^{2}-\bar{C}_{f} \frac{\left(1-\Lambda_{0} B / 2\right)^{2}}{1-\Lambda_{0} B} \Omega \times \\
\left\{1+\frac{\Omega^{2}}{\left(1-\Lambda_{0} B\right) \bar{v}_{b i}^{2}} \ln \left[1-\frac{\left(1-\Lambda_{0} B\right) \bar{v}_{b i}^{2}}{\Omega^{2}}\right]\right\}
\end{gathered}
$$

\section{NUMERICAL RESULTS AND THE COMPARISON WITH XHMGC}

\section{A. Methods and parameters}

Equation 13 is solved numerically for local dispersion relation. Equation 12 is solved for the symmetry breaking study of the central poloidal harmonics and compared with simulation results using XHMGC $\operatorname{code}^{9,28,29}$. For bell shape radial mode structures, the global solution is calculated using Galerkin method based on parabolic cylinder functions $h_{l}(r)$ as basis functions, which satisfy $\partial^{2} h_{l}(r) / \partial r^{2}+\left(2 l+1-r^{2}\right) h_{l}(r)=0$. The method adopted in this work is similar to that in [14] and is not explained in detail here.

Similar parameters as those in [19] are adopted. The safety factor profile is

$$
q(r)=q_{0}+\left(q_{a}-q_{0}\right)(r / a)^{2},
$$

where $q_{0}=1.9, q_{a}=2.3$. The energetic particle density profile is

$$
\begin{aligned}
& \bar{n}_{f}=\bar{n}_{f 0} C_{n f 0}\left\{1+C_{n f 1}\left[\tanh \left(\frac{r_{c f}-r}{r_{w f}}\right)-1\right]\right\}, \\
& \frac{d \bar{n}_{f}}{d r}=-\frac{\bar{n}_{f 0} C_{n f 0} C_{n f 1}}{r_{w f}}\left[1-\tanh ^{2}\left(\frac{r_{c f}-r}{r_{w f}}\right)\right],
\end{aligned}
$$

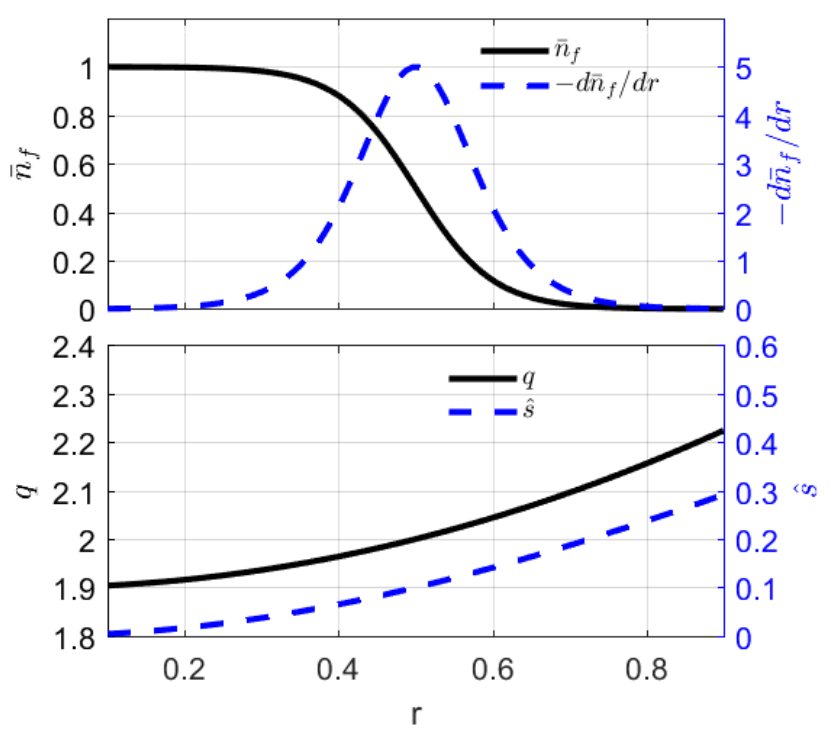

FIG. 1. Profiles of normalized energetic particle density $\bar{n}_{f}=$ $n_{f}(r) / n_{f}(r=0)$ and its radial derivative (left); profiles of safety factor $q$ and magnetic shear $\hat{s}$ (right).

where $C_{n f 0}=\left\{1+C_{n f 1}\left[\tanh \left(r_{c f} / r_{w f}\right)-1\right]\right\}^{-1} . \quad$ In simulation we use $C_{n f 1}=0.5, r_{c f}=0.5, r_{w f}=0.1$ and profiles are shown in Fig. 1. For the base case, other parameters at the reference radius are shown in Table I.

\section{B. Result benchmark for the base case}

The real frequency and growth rate calculated theoretically using the local approximation are shown in Fig. 2 and compared with XHMGC results. The agreement between the XHMGC results and theoretical results agree with each other reasonably. It should be noticed that the theoretical model in this work has the following approximations. The drive from $\partial F_{0 f} / \partial \epsilon$ is ignored due to the dominance of the $\partial F_{0 f} / \partial r$ term in $Q F_{0 f}$ (the ratio of the latter to the former is $k_{\theta} \rho_{f} q \omega_{t f} /\left(2 \omega \varepsilon_{n}\right) \gg$ $1)$; XHMGC uses slowing down EP distribution $F_{f}=$ $C_{0} \delta\left(\Lambda-\Lambda_{0}\right) /\left(\epsilon^{3 / 2}+\epsilon_{c}^{3 / 2}\right) H\left(\epsilon_{b}-\epsilon\right)$ (self consistently coupled to the field equation $\left.{ }^{29}\right)$, while the analytical model adopts the $\epsilon_{b} / \epsilon_{c} \gg 1$ approximation (Eq. 9) with $\beta_{f}$ matched to that in HMGC; the local eigenvalue is estimated at the mode rational surface; and the well passing particle assumption is adopted in the theoretical model while in XHMGC, particle motion is calculated according to equation of motion ${ }^{29}$. By using the analytical $K P C_{f}$, the technical complication is minimized but the key features due to EPs, e.g., the Hermitian and anti-Hermitian contribution from EPs, are kept. 


\begin{tabular}{c|c|c|c|c|c|c|c|c|c}
\hline$r_{c} / a$ & $a / R_{0}$ & $q_{c}$ & $T_{e} / T_{i}$ & $\rho_{i, c} / a$ & $v_{i, c} / v_{A, c}$ & $v_{b f, c} / v_{A, c}$ & $n_{f, c} / n_{i, c}$ & $\Lambda_{0} B$ & $v_{b f, c} / v_{c r i t, c}$ \\
\hline 0.5 & 0.1 & 2 & 0 & $0.002 \sqrt{2}$ & $0.06 \sqrt{2}$ & $0.3 \sqrt{2}$ & $0.006 / 2$ & 0 & $\sqrt{4.15}$ \\
\hline
\end{tabular}

TABLE I. Parametes for BAE (similar to those in [19]). The subscript 'c' denotes the value at the reference radius $r=r_{c}$. The $\sqrt{2}$ is due to the definition of $v_{t}=\sqrt{2 T / m}$ in this work rather than the XHMGC convention $\left(v_{t}^{X H M G C}=\sqrt{T / m}\right)$. $n_{f, c} / n_{i, c}=0.003$ corresponds to the on-axis value $n_{f, a x i s} / n_{i, c}=0.006$.

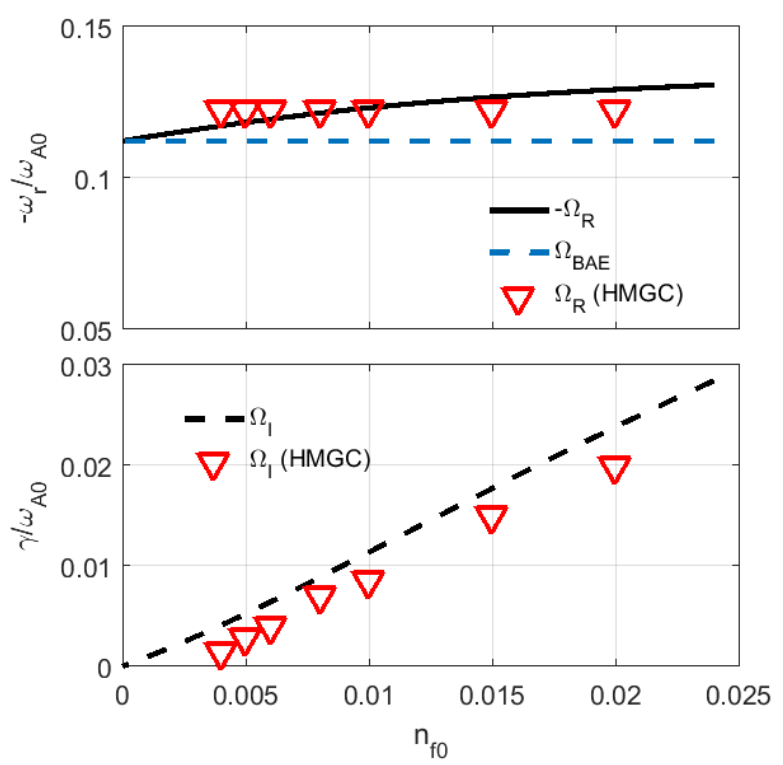

FIG. 2. Comparison of local solution and global solution from XHMGC. Note that $n_{f}\left(r=r_{c}\right)=n_{f 0} / 2$. XHMGC includes dissipation and the result exhibits a critical $n_{f 0}$ for growth rate $\gamma$ while the theoretical model ignores dissipation.

\section{The local and global solution with EP contribution}

The global eigenvalue and its connection to the local solution is shown in Fig. 3, where the local and global solutions are calculated using Eqs. 13 and 12 respectively. The local real frequency is mainly contributed by the kinetic particle compression term of thermal ions and the parallel field bending term $\Omega_{B A E}^{2}+(n q-m)^{2} / \beta_{i}$. The global growth rate is slightly lower than the largest local one (at $r \sim 0.5$ ). The global solution is a suitable superposition of local solutions with proper complex phases. The additional global correction due to the radial mode structure variation in the $\partial / \partial r$ terms of Eq. 12 leads to the change of the local eigenvalue and leads to the global eigenvalue formation.

\section{The parallel and radial symmetry breaking}

The parallel and radial symmetry breaking are two aspects of the global mode structure. The radial mode structure of the central poloidal harmonic and the con-

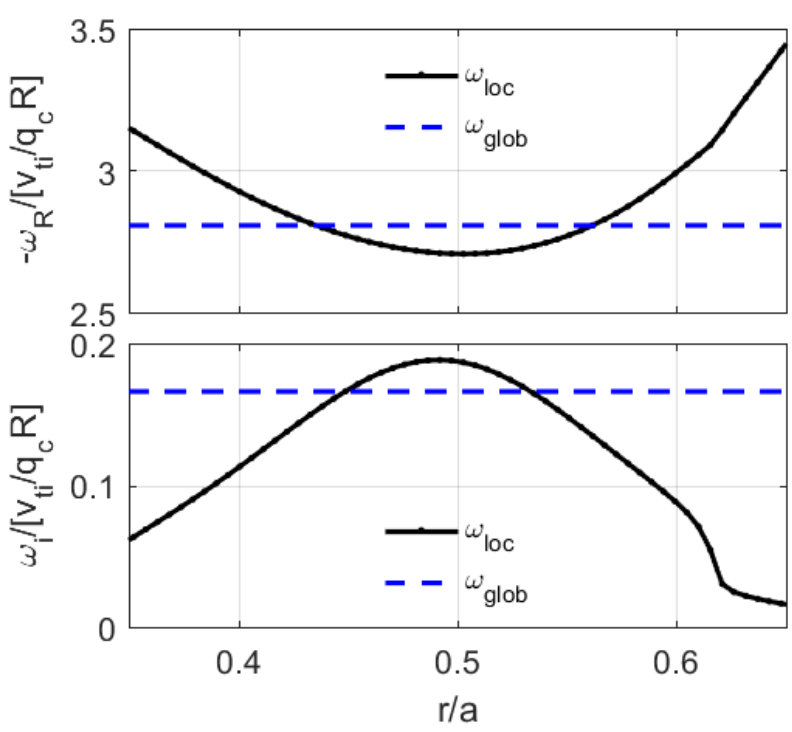

FIG. 3. Local and global eigenvalue of BAE in large aspect ratio limit. Compared with the local eigenvalue at $r / a=0.5$ $\left(\omega_{l o c}=-2.7062+0.1873 i\right)$, the global eigenvalue $\left(\omega_{\text {glob }}=\right.$ $-2.8065+0.1660 i$ ) has a growth rate around $12 \%$ lower than the local one and real frequency around $4 \%$ higher than the local one.

struction of the global 2D mode structure are shown in Fig. 4 for EP drive $\left(d n_{f} / d r\right)$ with different $r_{c f}$ values. For EP drive with $r_{c f}$ near $r / a=0.5$, the radial mode structure is relatively symmetric with respect to $r=r_{c f}$ (the central column). As $r_{c f} / a$ shifts away from 0.5, the radial mode structure symmetry breaking appears and the 2D "boomerang" structure is characterized with asymmetric tails (the left and right columns). The 2D mode structures obtained from XHMGC simulation support this point while the difference compared with the theoretical results can be from the simplifications in the theoretical model as mentioned in Section III B.

The radial symmetry breaking is analyzed as shown in Fig. 5. In these cases, the radial mode structure has a bell shape amplitude. The radial wave number $k_{r}$, defined according to $\delta \psi_{m}(r)=\exp \left\{i \int k_{r} d r\right\}$, describes the amplitude and phase variation along $r$. The maximum amplitude radial location is characterized by $\operatorname{Im}\left\{k_{r}\right\}=0$ (denoted by point $P_{I}$ ) and it overlaps with $\operatorname{Re}\left\{k_{r}\right\}=0$ (denoted by point $P_{R}$ ) for symmetric radial mode structure. The asymmetric radial mode structure is characterized by the mismatching between $P_{R}$ and $P_{I}$, which indicates a preferential propagation direction of the wave 

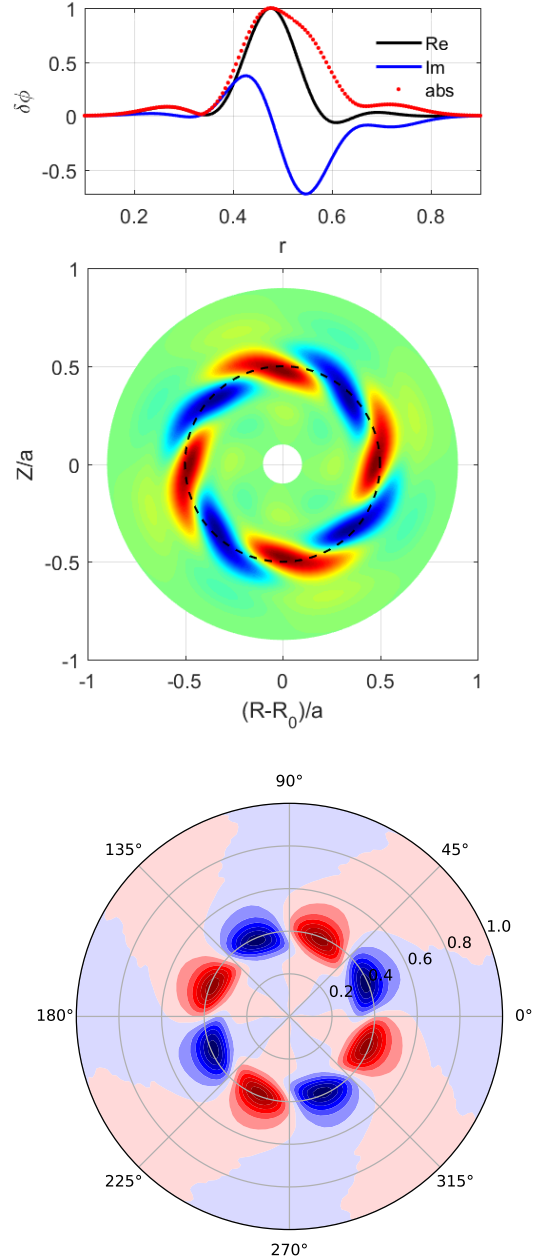
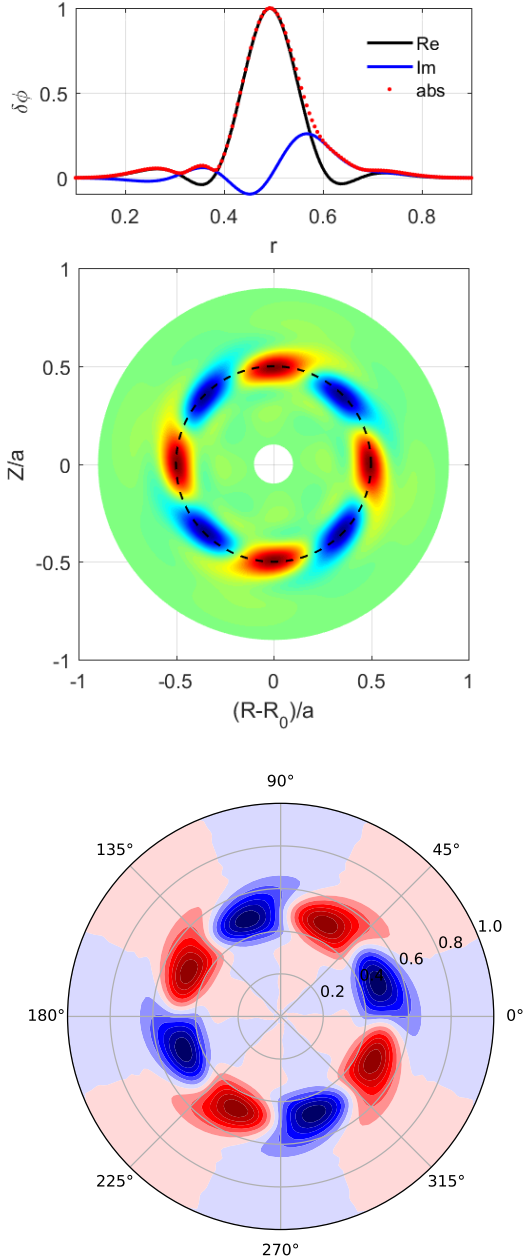
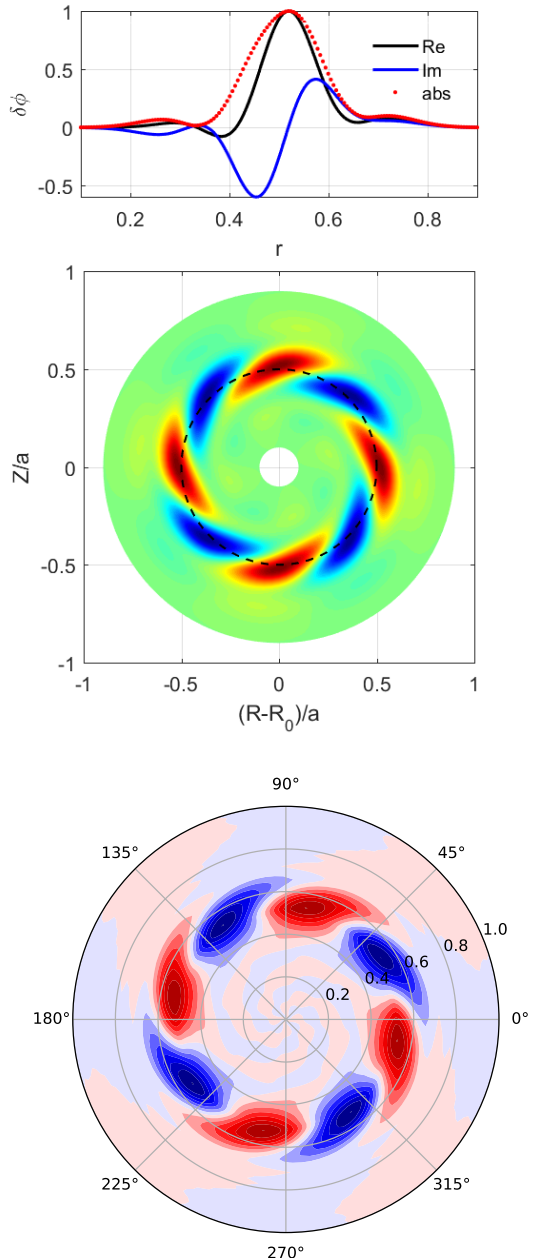

FIG. 4. Radial mode structure (first row) and 2D mode structure (second row) of BAE calculated theoretically for $r_{c f} / a=0.42$ (left), $r_{c f} / a=0.50$ (middle) and $r_{c} / a=0.55$ (right) with EP drive in large aspect ratio limit (Eq. 12 with the geometry effect term $1 / r \partial \delta \psi / \partial r$ ignored) Third row: $2 \mathrm{D}$ mode structure for $r_{c f} / a=0.42$ (left), $r_{c f} / a=0.50$ (middle) and $r_{c} / a=0.55$ calculated using XHMGC.

packet.

The parallel symmetry breaking in terms of the intensity weighted parallel $(n q-m)|\delta \psi|^{2}$ wave number is shown in Fig. 6, where $\delta \psi$ is the normalized perturbation with the maximum amplitude $|\delta \psi|=1$ at $r \sim 0.5$. Corresponding to the symmetric radial mode structure, the volume averaged $(n q-m)|\delta \psi|^{2}$, i.e., $\left\langle k_{\|}\right\rangle_{V}$, tends to vanish due to its opposite sign and similar magnitude inside and outside $r_{c f}$. As $r_{c f}$ shift away from the critical value for symmetric radial structure, the symmetry breaking of $(n q-m)|\delta \psi|^{2}$ appears and the volume averaged $(n q-m)|\delta \psi|^{2}$ can be generated. The generation of $\left\langle k_{\|}\right\rangle_{V}$ can be relevant to the wave-particle resonance and parallel residual stress/Maxwell stress and will be studied in the future.

The symmetry breaking in radial and parallel direction are intimately connected. Figure 7 shows the effect of the EP drive location on the symmetry breaking. As $r_{c f}$ shifts away from the critical value $r_{c f, 0} \sim 0.5 a$, the volume averaged $k_{r}$ and $k_{\|}$are generated and both increase in magnitude.

\section{DISCUSSION AND OUTLOOK}

The symmetry breaking of Beta-induced Alfvén Eigenmode (BAE) driven by energetic particles is studied based on theoretical model and simulation using $\mathrm{XH}-$ MGC. The theoretical formulae capture the important aspects of the radial and parallel symmetry breaking of the global mode structure and the non-perturbative effect of the energetic particles compared with the $\mathrm{XH}$ MGC simulation results. It is also shown that the radial and parallel symmetry breaking are intimately connected. The different types of distorted 2D mode structures can be produced depending on the radial location of the EP drive. It is also shown that the radial location 

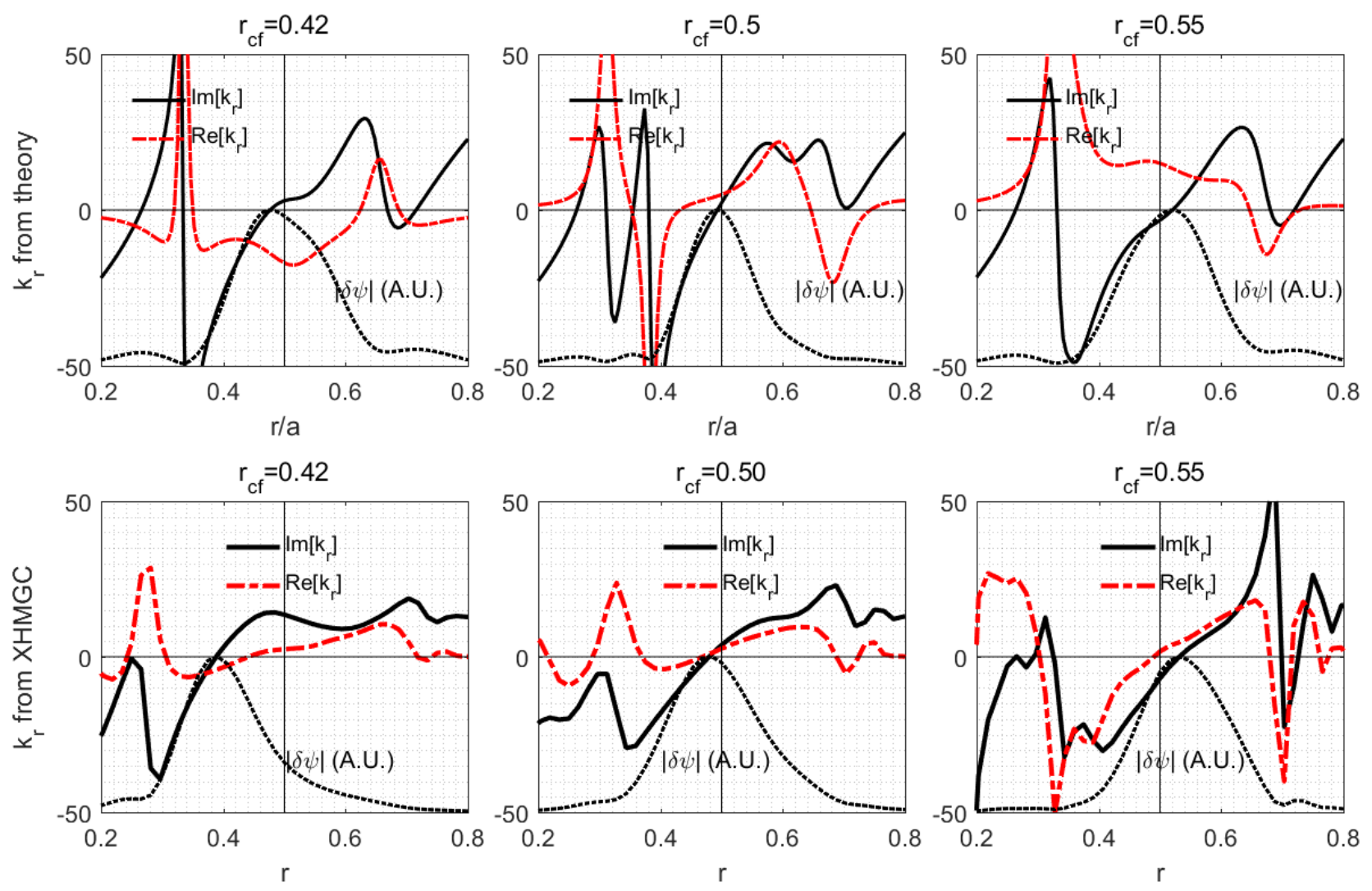

FIG. 5. Profiles of $|\delta \psi(r, n=2, m=4)|, k_{r, R}$ and $k_{r, I}$ for the base case (central column) and those with shifted EP drive (left and right columns). The mismatch between the $P_{R}$ point $\left(k_{r, R}=0\right)$ and $P_{I}$ point $\left(k_{r, I}=0\right)$ is observed for cases with shifted EP drive.

of the energetic particle drive is closely connected to the radial/parallel symmetry breaking and volume averaged radial/parallel wave number generation.

While this work focuses on the global analysis of the symmetry breaking, by using the analytical EP contribution and simplified geometry in theoretical model, more detailed analyses for parametric scaling and effects of different terms in the gyrokinetic quasi-neutrality and vorticity equations merit more efforts, by including more comprehensive physics and optimized numerical method. Several aspects as further steps are listed below.

1. While the present work ignored the FLR and FOW effects $\left(k_{\theta} \rho_{t i}=0.0226\right.$ for base case), the FLR and FOW effects is important for high $n$, high temperature plasma, in particular, when energetic particles are present. While previous work on microturbulence symmetry breaking observed that the competition between the FLR term and magnetic drift term can lead to the $k_{\|}$reversal $^{30-32}$, the FLR and FOW effects of thermal ions and EPs for BAE symmetry breaking are not clear. The consideration of FLR and FOW effects, either in theoretical analyses, XHMGC simulation or the more comprehensive eigenvalue code LIGKA $^{7}$, can be important for general cases in both local and global analyses.
2. While the BAE with bell shape radial structure is observed from XHMGC simulation a priori for the present chosen parameters, the critical condition for the BAE excitation such as critical EP and thermal ion parameters needs to be analyzed in detail, by comparing effect of thermal ion kinetic and EP's Hermitian and anti-Hermitian contributions. The finite element method ${ }^{7,33}$ or finite difference method ${ }^{34}$ will be useful to identify the transition from the singular radial structure solution to smooth one and the corresponding critical excitation condition.

3 . The connection between the present BAE problem with monotonous $q$ profile to the Alfvén eigenmode or energetic particle mode (EPM) for hollow $q$ profiles $^{33,35,36}$ and toroidal induced Alfvén mode $^{18}$ can be analyzed considering the similarity in the equation structure with kinetic effects. The consideration of the non-perturbative EP Hermitian and anti-Hermitian contribution can be important in the study of mode width and symmetry features.

The global linear study in this work can shed light on the understanding of nonlinear simulation. During non- 

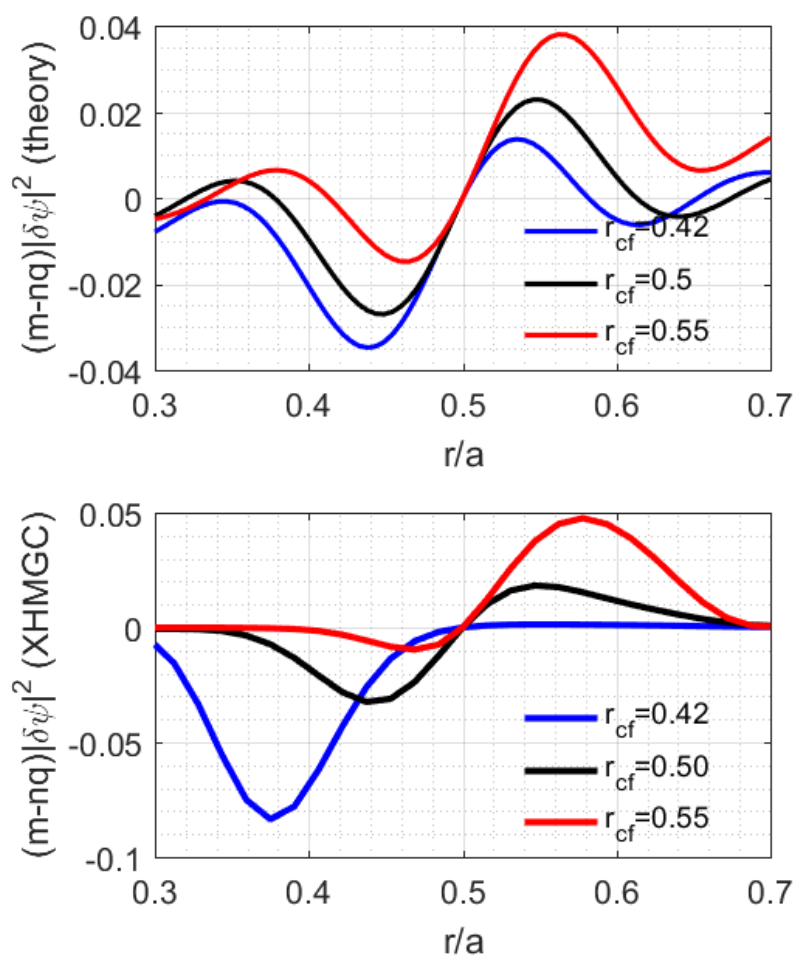

FIG. 6. Radial profiles of $(m-n q)|\delta \psi|^{2}$ calculated theoretically (upper) and from XHMGC (lower). Only the central poloidal harmonic is kept. It is indicated that volume averaged $k_{\| \mid}|\delta \psi|^{2}$ is generated as EP drive center $r_{c f}$ shifts away from BEA accumulation point.

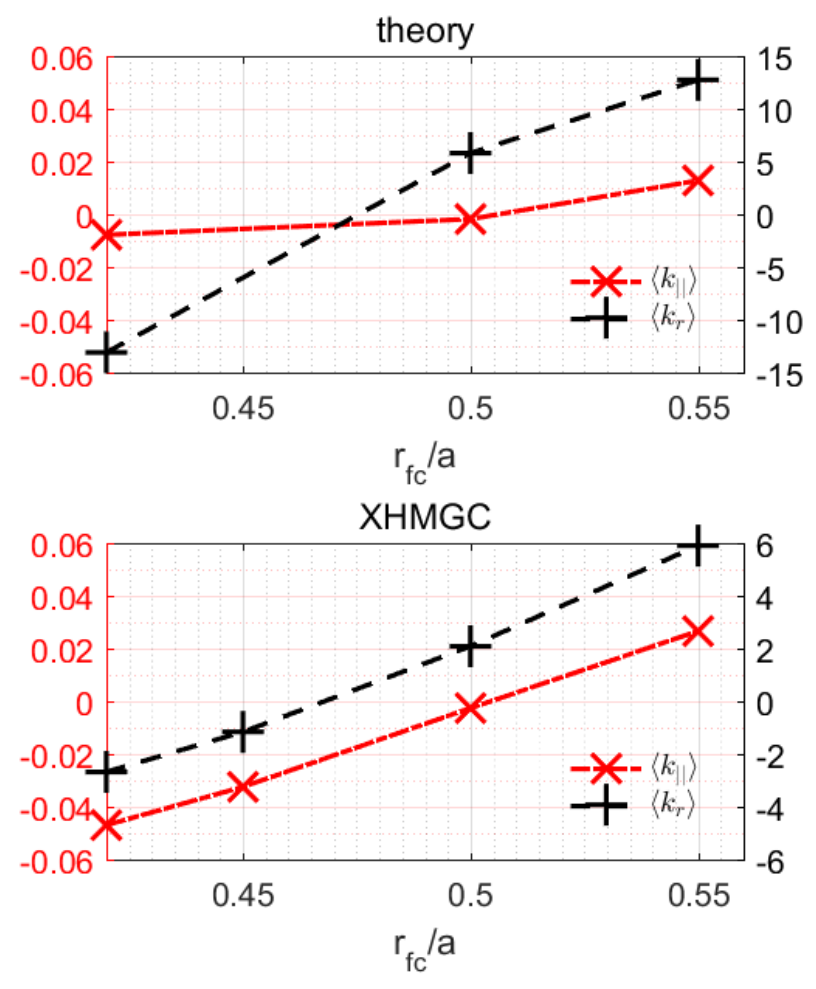

FIG. 7. Volume averaged $k_{r}$ and $k_{\|}$for different EP drive locations $r_{c f}$. linear stage, the EP density profile is flattened and the drive center shifts inward or/and outward. As observed in this work, the mode structure is modified due to the EP drive center shift. Then the symmetry breaking in terms of $k_{\|}$can enter the wave-particle resonance condition and in turn can change the EP driven instabilities. Developing the diagnosis tool in nonlinear simulation to track the mode structure can be helpful for identifying the interplay of EP transport and instability excitation.

The study of the non-pertubative EP effect and symmetry breaking can be important for the future burning plasma considering the higher EP portion and energy compared with the present ones. Besides the mode structure symmetry breaking study, its relevance to other problems can be analyzed in the future, such as the effects of the symmetry breaking on the wave-particle interaction between EPs/thermal ions and Alfvén eigenmode and the consequent thermal ion momentum transport ${ }^{5,6}$ and EP transport ${ }^{4}$.

\section{ACKNOWLEDGMENT}

Discussion with A. Bierwage, I. Chavdarovski, O. Maj and L. Chen and computing/software assistance from T. Hayward-Schneider are appreciated by Z. X. Lu. Support from NLED and NAT projects is acknowledged. This work has been carried out within the framework of the EUROfusion Consortium and has received funding from the Euratom research and training programme 2014-2018 under grant agreement No 633053. The views and opinions expressed herein do not necessarily reflect those of the European Commission.

${ }^{1}$ F. Zonca and L. Chen, Phys. Plasmas 21, 072120 (2014).

${ }^{2}$ F. Zonca and L. Chen, Phys. Plasmas 21, 072121 (2014).

${ }^{3}$ L. Chen and F. Zonca, Rev. Modern Phys. 88, 015008 (2016).

${ }^{4}$ W. Zhang, Z. Lin, and L. Chen, Phys. Rev. Lett. 101, 095001 (2008).

${ }^{5}$ Y. Camenen, Y. Idomura, S. Jolliet, and A. Peeters, Nucl. Fusion 51, 073039 (2011).

${ }^{6}$ A. G. Peeters, C. Angioni, A. Bortolon, Y. Camenen, F. J. Casson, B. Duval, L. Fiederspiel, W. A. Hornsby, Y. Idomura, T. Hein, et al., Nucl. Fusion 51, 094027 (2011).

${ }^{7}$ P. Lauber, S. Günter, A. Könies, and S. D. Pinches, J. Compu. Phys. 226, 447 (2007).

${ }^{8}$ Y. Todo, H. Berk, and B. Breizman, Nucl. Fusion 50, 084016 (2010).

${ }^{9}$ S. Briguglio, G. Vlad, F. Zonca, and C. Kar, Phys. Plasmas 2, 3711 (1995).

${ }^{10}$ F. Zonca and L. Chen, Phys. Rev. Lett. 68, 592 (1992).

${ }^{11}$ Z. X. Lu, F. Zonca, and A. Cardinali, Phys. Plasmas 19, 042104 (2012).

12 R. Ma, F. Zonca, and L. Chen, Phys. Plasmas 22, 092501 (2015).

${ }^{13}$ F. Zonca, L. Chen, and R. A. Santoro, Plasma Phys. Controlled Fusion 38, 2011 (1996).

${ }^{14}$ Z. X. Lu, E. Fable, W. Hornsby, C. Angioni, A. Bottino, P. Lauber, and F. Zonca, Phys. Plasmas 24, 042502 (2017).

${ }^{15}$ W. Heidbrink, M. Van Zeeland, M. Austin, E. Bass, X. Chen, B. Grierson, C. Muscatello, G. Matsunaga, G. McKee, D. Pace, et al., in Proc. 24th Int. Conf. on Fusion Energy (San Diego, $C A$, 2012) (2012).

${ }^{16}$ Y. Liu, Z. Lin, H. Zhang, and W. Zhang, Nucl. Fusion 57, 114001 (2017). 
${ }^{17}$ L. Chen and F. Zonca, Phys. Plasmas 24, 072511 (2017).

${ }^{18}$ Z. Wang, Z. Lin, I. Holod, W. Heidbrink, B. Tobias, M. Van Zeeland, and M. Austin, Phys. Rev. Lett. 111, 145003 (2013).

${ }^{19}$ X. Wang, S. Briguglio, P. Lauber, V. Fusco, and F. Zonca, Phys. Plasmas 23, 012514 (2016).

${ }^{20}$ H. Cai, S. Wang, Y. Xu, J. Cao, and D. Li, Phys. Rev. Lett. 106, $075002(2011)$.

${ }^{21}$ N. Gorelenkov, C. Cheng, and W. Tang, Phys. Plasmas 5, 3389 (1998).

${ }^{22}$ J. Cheng, W. Zhang, Z. Lin, I. Holod, D. Li, Y. Chen, and J. Cao, Phys. Plasmas 23, 052504 (2016).

${ }^{23}$ H. Zhang, Y. Liu, Z. Lin, and W. Zhang, Phys. Plasmas 23, 042510 (2016).

${ }^{24} \mathrm{~L}$. Chen and A. Hasegawa, Journal of Geophysical Research: Space Physics 96, 1503 (1991).

${ }^{25}$ P. Lauber, M. Brüdgam, D. Curran, V. Igochine, K. Sassenberg, S. Günter, M. Maraschek, M. García-Muñoz, N. Hicks, and the ASDEX Upgrade Team, Plasma Phys. Controlled Fusion 51, 124009 (2009).
${ }^{26}$ H. S. Zhang, Z. Lin, I. Holod, X. Wang, Y. Xiao, and W. L. Zhang, Phys. Plasmas 17, 112505 (2010).

${ }^{27}$ S.-T. Tsai and L. Chen, Phys. Fluids B 5, 3284 (1993).

${ }^{28}$ S. Briguglio, F. Zonca, and G. Vlad, Phys. Plasmas 5, 3287 (1998).

${ }^{29}$ X. Wang, S. Briguglio, L. Chen, C. Di Troia, G. Fogaccia, G. Vlad, and F. Zonca, Phys. Plasmas 18, 052504 (2011).

${ }^{30}$ Z. X. Lu, Phys. Plasmas 22, 052118 (2015).

${ }^{31}$ Z. X. Lu, W. Wang, P. Diamond, G. Tynan, S. Ethier, J. Chen, C. Gao, and J. Rice, Nuclear Fusion 55, 093012 (2015).

${ }^{32}$ Z. X. Lu, W. X. Wang, P. Diamond, G. Tynan, S. Ethier, C. Gao, and J. Rice, Phys. Plasmas 22, 055705 (2015).

${ }^{33}$ G. Fu and H. Berk, Phys. plasmas 13, 052502 (2006).

${ }^{34}$ H. S. Xie, Y. Y. Li, Z. X. Lu, W. K. Ou, and B. Li, Phys. Plasmas 24, 072106 (2017)

${ }^{35}$ F. Zonca, S. Briguglio, L. Chen, S. Dettrick, G. Fogaccia, D. Testa, and G. Vlad, Phys. Plasmas 9, 4939 (2002).

${ }^{36}$ H. Berk, D. Borba, B. Breizman, S. Pinches, and S. Sharapov, Phys. Rev. Lett. 87, 185002 (2001). 